\title{
COMPREHENSIVE REHABILITATION IN CONSERVATIVE METHOD OF TREATMENT OF PATIENTS WITH RARE DISEASE ARTHROGRYPOSIS MULTIPLEX CONGENITA
}

\author{
M. Staneva*, K. Mollova, A. Uzunova \\ Medical College, Trakia University, Stara Zagora, Bulgaria
}

\begin{abstract}
Introduction. Arthrogryposis is a genetic disorder characterized by muscle imbalance, contractures and deformation of the joints of the limbs. The abilities for passive and active movements are very limited. The disease is associated with other diseases and syndromes.

After performing functional diagnostics, the rehabilitation proceeds with a therapeutic plan created beforehand for conservative treatment depending on the health needs of the patient.

The purpose of this paper is to examine the literature sources and achievements in practice and their application in complex rehabilitation.

Methods. Literature research; a carefully selected physiotherapy, massage and kinesiotherapy procedures for better mobility and opportunity for self-service of patients with arthrogryposis.

Conclusion. The prolonged implementation of the comprehensive rehabilitation and hard work of the team have a good therapeutic effect and contribute significantly to improving the quality of life of the sick child.
\end{abstract}

Key words: arthrogryposis, diagnostics, conservative method, rehabilitation, quality of life.

\section{INTRODUCTION}

Arthrogryposis, also known as arthrogryposis multiplex congenita gets its name from the Greek and literally means "curved or crooked joints." This is a rare inherited disease that is characterized by contractures and deformation of the joints of the limbs (Figure 1). The cause of this disease is fetal akinesia or reduced fetal movements, due to neurogenic, muscle or connective tissue abnormalities, and mechanical movement restrictions. Joint contractures are detected at birth. Affected limbs are distorted, poorly expressed subcutaneous fat tissue, without skin folds. Deformities are usually symmetrical and distal, the hands and feet are most severely affected. (1) Typical is the position of the body

\footnotetext{
*Correspondence to: Mariana Staneva, e-mail: mariqna_60@abv.bg,tel.:00359887307398,9 Armeiska Str., Stara Zagora 6000, Bulgaria
}

at birth - curved and adducted shoulders, fixed in extension elbows, pronation of forearms, fixed wrists and fingers. The legs are in severe clubfoot (Figure 2). The muscle abnormalities form after a time - reduced muscle mass, fibrous adhesions, soft texture, and abnormal connection of tendors. The end result is muscle shortening. In turn this defect leads to another complication - it impairs the ability to perform active and passive movements in the affected joints. Arthrogryposis is not a separate disease, but usually occurs in a relation with other diseases and syndromes, but rarely on its own. The most common problems combined with joint arthrogryposis are pulmonary hypoplasia, cryptorchidism, congenital heart malformations, tracheoesophageal fistula, and inguinal hernia, visual or ocular anatomical disorders. In most cases, is not inherited and does not occur more than once in a family. The intellect is retained. (2) 


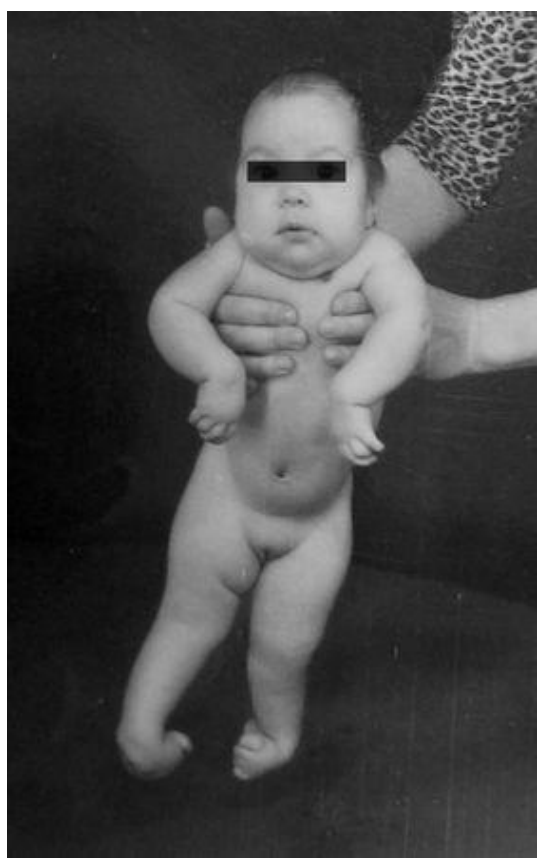

Figure 1. Child with arthrogryposis

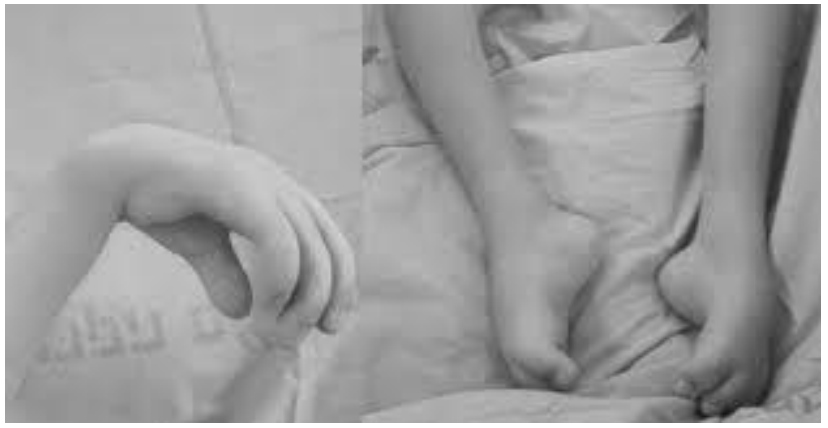

Figure 2. Affected distal parts with arthrogryposis

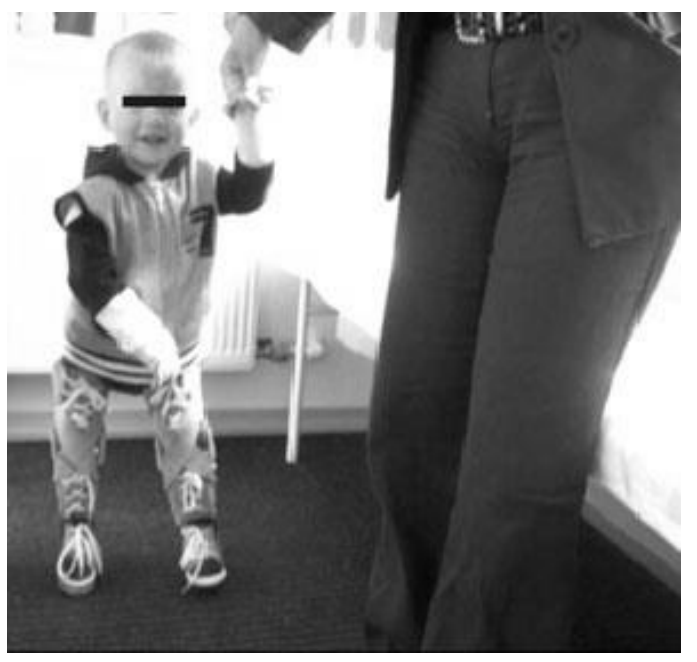

Figure 3. Arthostatic means

The purpose of this paper is to provide the means of kinesitherapy and combining them with physical factors and therapeutic massages to optimize the methodology of complex rehabilitation, based on literature research and work with a patient diagnosed with arthrogryposis.

\section{MATERIYAL AND METHODS}

Conducted is a literature research in the field of physical therapy related to the arthrogryposis disease and the relevant menas and techniques have been applied in the complex rehabilitation of the patient.

A complex rehabilitation is conducted of a patient diagnosed with arthrogryposis in outpatient care in Stara Zagora.

\section{RESULTS AND DISCUSSION}

Based on the conducted research it became clear that the treatment of arthrogryposis contractures and deformities is a complicated and a lengthy problem in the complex treatment. Controversial is the question of the sequence in the eliminating the distortions of the joints in their complex elimination. Some experts suggest corrections beginning with the distal joints and others with the proximal and straightening the axes of the limbs. (3)

Treatment For upper limbs is aimed at creating conditions for self-service and for the lower limbs - at the restoration of locomotor function.

A consistent treatment plan is made including conservative and operative method, the implementation of which will improve the quality of life of the patient. (4)

A system for early conservative treatment of children with arthrogryposis is created that includes kinesitherapy, massage, physical therapy, orthotics and others.

The treatment is symptomatic, long, hard and in stages, it begins with birth and is determined by the occured changes. The early period of child's life is the most favorable as cartilage in the newborn is the most elastic and then the development of the motor skills begins. (5)

The selection of methods and means in the complex treatment comply with the age and functionality of the sick child.

When working with the patient during the conduct of several rehabilitation courses there are various physical means used aimed at 
STANEVA M., et al.

eliminating distortions and contractures, development and maintenance of the active function for preserving the muscle tissue and for a maximum retention of the limbs in the corrected position.(6)

\section{Physiotherapy complex includes:}

- Electro stimulation with suitable pulse according to the electro diagnosis of muscles showing the lowest capacity;

- Low frequency magnetic field;

- Medium frequency current on the distal extremities;

- Heat therapy and compress with lye.

The therapeutic massage begins 2-3 weeks after childbirth. A massage of arms and legs is performed, emphasizing on the joints with contractures, then a massage of the back and the gluteal muscles is carried out. The massage is done briefly and selectively - for the tight muscles gently and slowly, and for the weak muscles more vigorously. (7)

\section{Kinesitherapeutic complex includes:}

- Special reflective exercises;

- Passive exercises of soft tissue;

- Methods of Bobath and Vojta;

- Underwater gymnastics;

- Active exercises.

\section{CONCLUSION}

The treatment for multiple deformities in arthrogryposis is a long and labor-intensive process and requires perseverance by both the therapists and by the child and parents. Based on carefully selected physiotherapy, massage and kinesiotherapy procedures there have been created conditions for better mobility and opportunity for self-service of the patients with arthrogryposis.(8)

Despite the absence of full recovery of limb function, the persistent and systematic rehabilitation gives good results.

The most important objective of the whole team of therapists is to provide independent life of patients with arthrogryposis. (9)

\section{REFERENCES}

1. Gatev St., St. Bankov, St. Busarov, Manual in Physical Therapy, Sports and Medicine, Volume 2, Sofia, 1992.

2. Malakhov O., I. Kosov, I. But-Gusaim, S. Mihailova, Clinic, diagnosis and treatment of arthrogryposis in children / Journal of Trauma Orthopaedics, Issue 2, pp. 79-86, 2003.

3. http://www.puls.bg/health/children/news_1 5708.html

4. http://www.specialchild.com/archives/dz036.html

5. http://www.turner.ru/sys_artogrip.html

6. http://www.hapche.bg/meditsinskirechnik/arthrogriposis

7. http://ivocholakov.alle.bg

8. http://www.ilyssamed.ru/pages/artrogripoz

9. http://www.arthrogryposis.de/iga/info_ru 